\title{
A Case of Engorged Female Hard Tick in the External Auditory Canal of an Infant
}

\author{
Woo-Jung Sung', Yee-Hyuk Kim²,* \\ 'Department of Pathology, ${ }^{2}$ Department of Otorhinolaryngology-Head \& Neck Surgery, School of Medicine, Catholic University of Daegu, \\ Daegu 42472, Korea
}

\begin{abstract}
An oval-shaped mass with a smooth surface was found in the ear canal of a 22-month-old male infant. Although the mass appeared to be almost entirely blocking the ear canal, it was successfully removed under a surgical microscope without general anesthesia at the outpatient department. Under an optical microscope with hematoxylin and eosin staining, the specimen was observed to have a cuticle with a serrated surface and a pore canal, as well as parts of the capitulum, salivary glands, muscles, midgut, and the legs. The specimen was identified as a hard tick of the family Ixodidae, based on gross and histological findings. This paper is the first report in Korea on the diagnosis and treatment of a tick bite in the ear canal.
\end{abstract}

Key words: Infant, ear canal, tick

\section{INTRODUCTION}

Serious illnesses, such as Lyme disease or severe fever with thrombocytopenia syndrome (SFTS), can occur in people who are bitten by ticks $[1,2]$. Therefore, after a bite occurs, it is very important to remove the tick from the human body as soon as possible [3]. However, in a geographical region or country like Korea, where ticks are rare, the diagnosis of a tick bite might not be readily confirmed, even when an engorged tick is discovered upon physical examination $[4,5]$. In particular, a true tick bite in humans occurs mainly in the head and neck region, on the arms and/or on the legs $[1,2,6]$. Tick bites rarely involve the ear canal, which makes it difficult to diagnose actual cases of tick bites in the ear canal. Based on a review of past publications, it is evident that there has not been a single reported case of tick bite involving the ear canal in Korea. The current paper thus represents the first case in Korea involving the diagnosis and treatment of a tick bite in the ear canal.

\footnotetext{
- Received 9 June 2017, revised 17 August 2017, accepted 24 August 2017.

*Corresponding author (yhukim@cu.ac.kr)

(c) 2017, Korean Society for Parasitology and Tropical Medicine

This is an Open Access article distributed under the terms of the Creative Commons Attribution Non-Commercial License (http://creativecommons.org/licenses/by-nc/4.0) which permits unrestricted non-commercial use, distribution, and reproduction in any medium, provided the original work is properly cited.
}

\section{CASE RECORD}

A 22-month-old male infant visited an external hospital (private otolaryngologist) for symptoms of cold in April 2015. At this time, an ear canal examination was performed to evaluate the otitis media. During this examination, the otolaryngologist incidentally found a suspected epidermoid cyst in the right external auditory canal, and the patient was referred to the outpatient otolaryngology clinic of our hospital for further evaluation and treatment. The mass suspected to be an epidermoid cyst in the right external auditory canal was removed with alligator forceps under a surgical microscope, and was sent to pathology for histological examination (Fig. 1). The parents reported that the infant's newborn hearing screening test at birth was normal, and that the infant had passed the test for both ears. Following the removal of the mass from the external ear canal, the right eardrum appeared normal on endoscopic examination. The result of histological examination 1 week later revealed the presence of a hard tick. On microscopic examination, the specimen was found to be an ovalshaped hard tick with a width of $4 \mathrm{~mm}$ and a length of 5.5 $\mathrm{mm}$ (Fig. 2A). We also observed a cuticle surrounding the outermost edge, a serrated surface, and a pore canal (Fig. 2B). Parts of the capitulum (Fig. 2C), salivary glands (Fig. 2D), and muscles were also observed. A partially damaged midgut was observed on the side, and the tick's legs were observed on the 

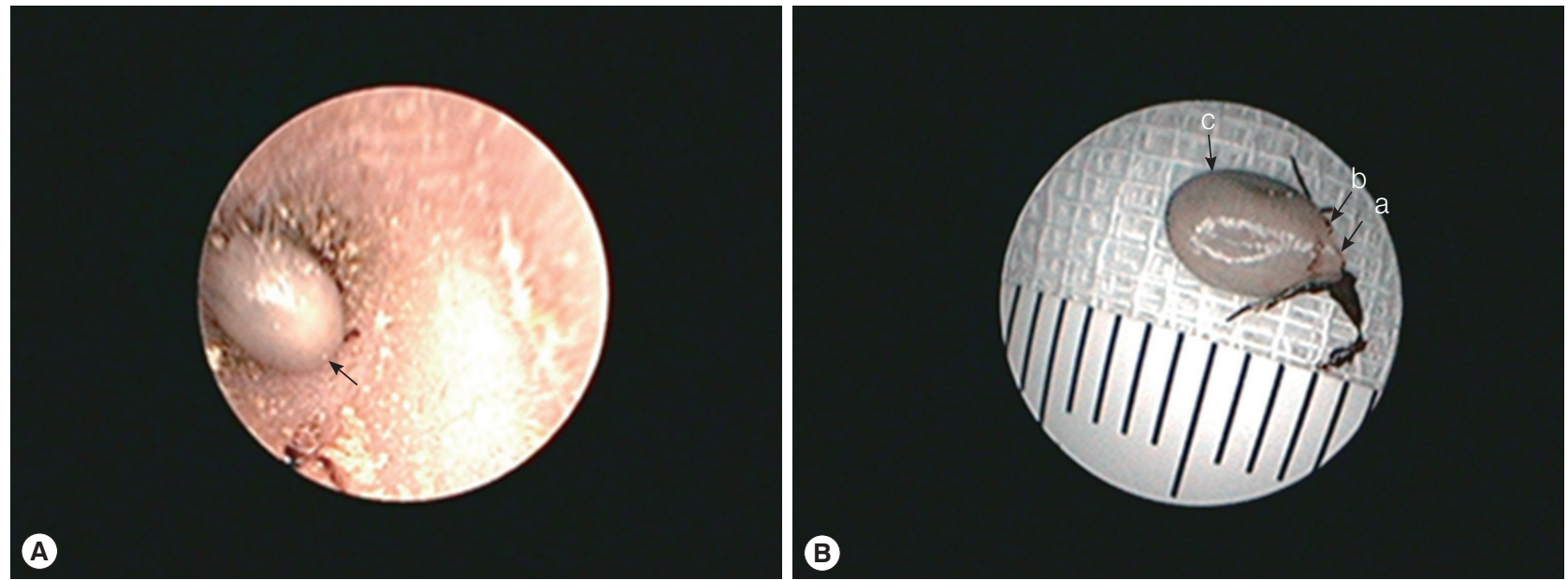

Fig. 1. Photographs of the tick observed under an ear endoscope. (A) The engorged tick (arrow) feeding from the external auditory canal was almost completely blocking the external auditory canal in this patient. (B) The tick was removed from the external auditory canal. The length of the body was approximately $5.5 \mathrm{~mm}$, and it was oval-shaped. The scutum (a), scutum posterior margin (b), and alloscutum (c) were observed in its body.
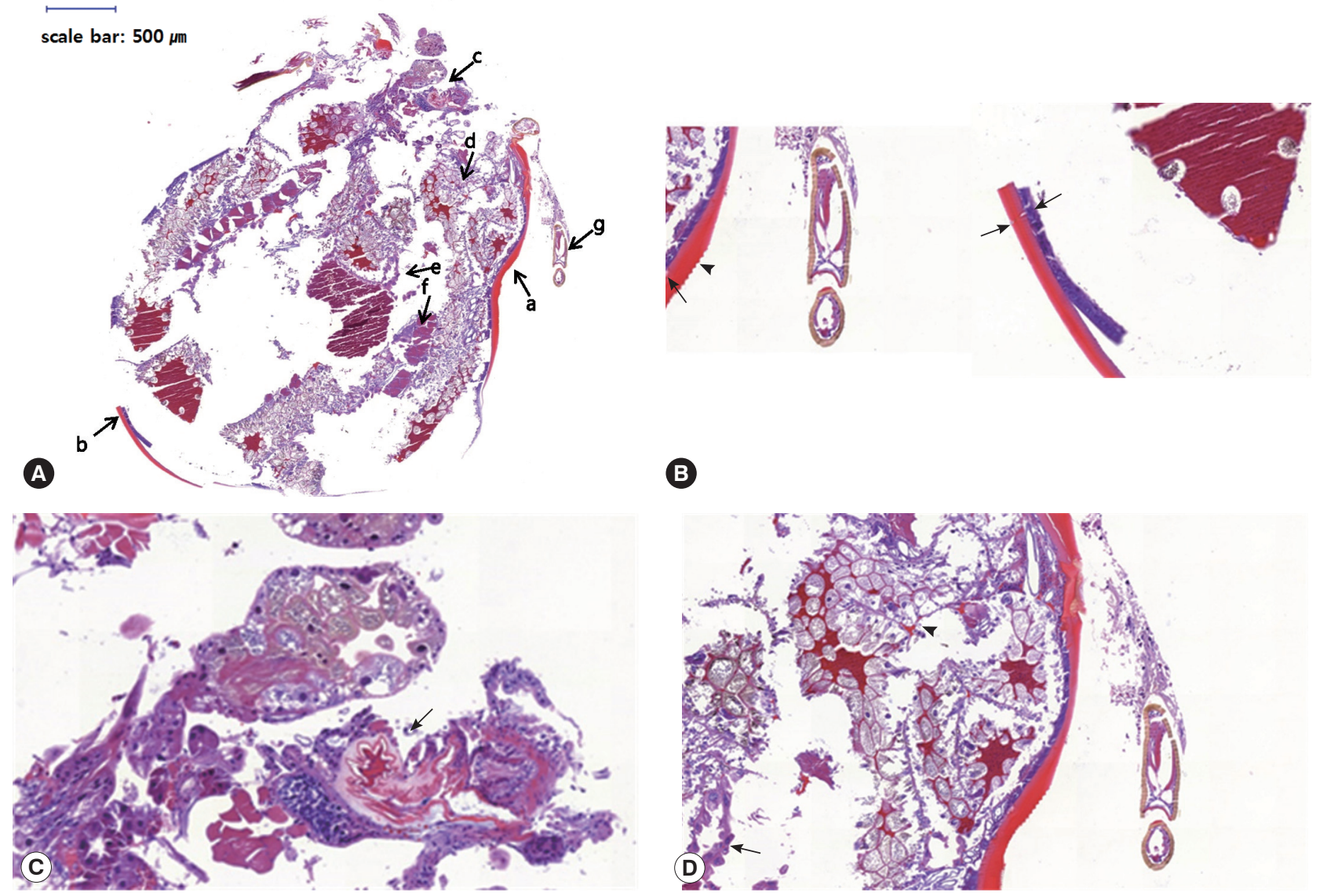

B

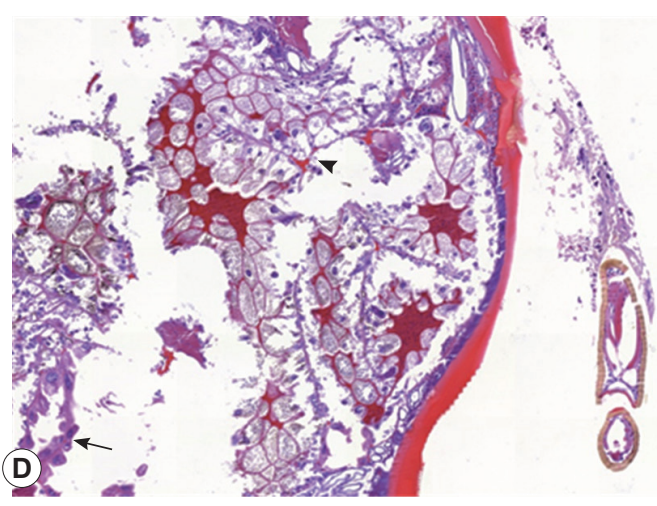

Fig. 2. Histological findings of the tick removed from the external ear canal. (A) The cuticle (a, b), parts of the capitulum (c), salivary gland (d), midgut (e), musculature (f), and leg (g) were observed in the whole section of the tick. (B) The pore canal (arrows) of the cuticle and its saw-like surface (arrowhead) were observed in the zoomed-in images of 'a' and 'b' (HE stain, $\times 200)$. (C) A part of the capitulum (arrow) was observed in the zoomed-in image of 'c' (HE stain, $\times 200)$. (D) A salivary gland (arrowhead) and midgut (arrow) were observed in the zoomed-in images of 'd' and 'e' (HE stain, $\times 100)$. 


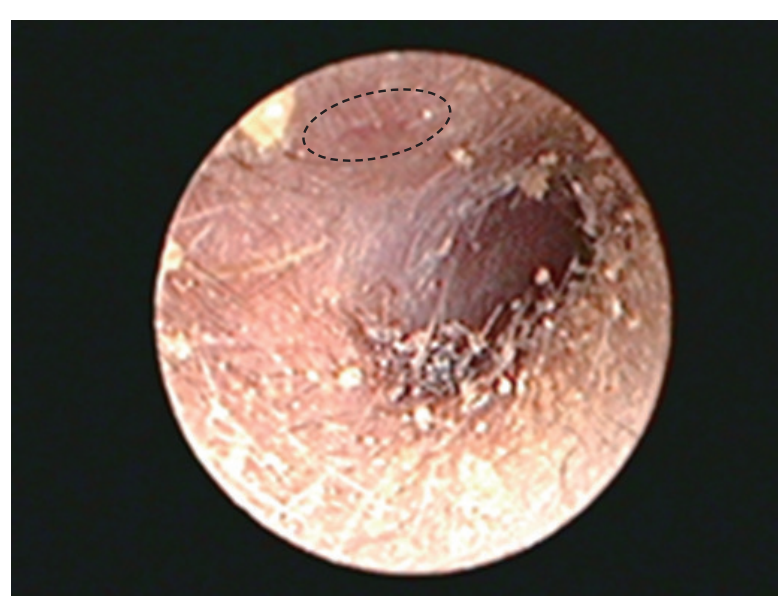

Fig. 3. The dashed circle indicates the area of the external ear canal affected following tick removal. Three weeks after the tick removal, slight erythema was observed at the site. However, it was deemed to be a healing process.

outside of the body (Fig. 2A). The specimen was diagnosed as a hard tick of the family Ixodidae, based on the gross and histological findings, but the genus and species of the tick could not be determined.

On the first day of the 1-week follow-up period, ear canal endoscopy showed that a crust had formed in the area of the right external auditory canal from where the tick was removed. The tick's mouthparts remained embedded in the skin, and had been covered by the crust. The mouthparts were removed, and treatment with antibiotics was prescribed. Although Dae$\mathrm{gu}$, one of the largest cities in Korea, was home to the patient, the patient mostly resided at his grandparent's house in a suburban area that is approximately $100 \mathrm{~km}$ from Daegu. The patient's grandparent had a dog in the front yard of the house, and the patient often played on the hills and meadows near the house. During the third week of follow-up, slight erythema was observed in the right ear canal at the site at which the tick had been removed from, but this was considered to be a part of the normal healing process (Fig. 3). During the eighth week, there were no abnormal findings in the right external auditory canal, and the patient had not developed any systemic symptoms, including fever. At the time of the final physical examination, the patient's body temperature was normal, and there were no local symptoms, such as skin rash, observed.

\section{DISCUSSION}

Ticks attach to the skin of vertebrates and feed upon body fluids, such as blood and lymph [2]. The alloscutum of the swollen female tick, which is inflated during feeding, appears as a smooth gray oval shape with a size of approximately 5-10 $\mathrm{mm}$, and is similar in appearance to an epidermoid cyst [7]. In this case, the engorged female tick, which was initially suspected to be an epidermoid cyst, was $5.5 \mathrm{~mm}$ in length, which, though not extremely large, was still sizable enough to almost entirely block the external auditory canal of the patient. As noted, the engorged female tick in this case was similar in shape to an epidermoid cyst. However, while epidermoid cysts typically have a broad base at the point of contact with the skin and a low (in terms of their vertical height from the base) dome shape, the engorged female tick in question had a relatively narrow base of contact with the skin of the external auditory canal, and was taller (in terms of its anteroposterior length). Moreover, the surface of the typical epidermoid cyst is soft and tender, while that of the engorged female tick was relatively firm. These characteristics could provide a differential clue to suggest the presence of an engorged tick during a physical examination.

Ticks become active as early as March, and their activity peaks between May and August. Thus, there is a higher likelihood that tick bites can occur during these months. The tick bite reported in this case occurred in April, which fits the timeframe of known high tick activity. Ticks are very slow in action, and tend to climb onto grassy leaves in a forest to wait for the passage of animals or people, rather than outright chasing a potential host [2]. Tick bites are frequent in people attending picnics, or those with periodic exposure to plants, such as farmers [6]. Furthermore, it has been reported that ticks are often found in domestic dogs, as well as in livestock such as cattle and horses; domestic dogs can become carriers of tick vectors to humans $[1,6,8]$. The child in this case report seems to have acquired his tick bite via a bush nearby, or from the grandparent's dog.

Cases involving a hard tick being present in the external auditory canal have been reported only rarely in industrialized countries such as Korea $[1,9]$. The species that could be identified in these cases were Ixodes ovatus and Dermacentor variabilis, and were thought to have been transferred from pet dogs $[1,9]$. In industrialized countries, there is generally less contact with crops and cattle, but more frequent physical contact with pet animals, and this is thought to be the cause of most tick bites. Adolescent and adult patients with a tick bite in the external auditory canal may present with symptoms of aural fullness, pruritus, crackling sensation, tinnitus, and otalgia $[1,3,9,10]$, 
but in infants, who are unable to express or convey these sensations, ticks are sometimes discovered only by chance, like in the present case. Facial palsy is one of the more severe symptoms that can arise due to a tick bite in the external auditory canal or on the tympanic membrane, and this is thought to be the result of neurotoxins secreted from the salivary glands of ticks $[3,10]$.

The external auditory canal is not an open space like the neck, arms, or scalp is, but rather a narrow, tube-shaped space. As in this case, infants do not always cooperate with procedures (not lying still while removing the tick), so general anesthesia is commonly used. However, the parents in the current case did not want their child to undergo general anesthesia. As a general precaution when removing ticks, no pressure should be applied to the abdomen of the tick, to prevent transmission of tick-borne pathogens, and any mouthparts embedded under the host's skin should be removed to prevent secondary infection $[1,5]$. Alligator forceps were inserted between the external auditory canal and the tick in this case, and everything except the mouth part of the tick, which was observable in the external auditory canal, was removed at first try, and was subsequently followed with the removal of the mouthpart embedded under the external auditory canal at 1 week later. This method was easy to incorporate in a narrow ear canal and, due to the short treatment time, general anesthesia was not required even though the patient was an infant. With this method, we were able to completely remove the mouthpart without applying pressure to the abdomen of the tick.

\section{CONFLICT OF INTEREST}

All authors declare no conflict of interest.

\section{REFERENCES}

1. Iwasaki S, Takebayashi S, Watanabe T. Tick bites in the external auditory canal. Auris Nasus Larynx 2007; 34: 375-377.

2. Shin EH. Hard tick species including SFTS-infected ticks and bite cases in the Republic of Korea. Public Health Weekly Report, KCDC 2014; 7: 342-345.

3. Patil MM, Walikar BN, Kalyanshettar SS, Patil SV. Tick induced facial palsy. Indian Pediatr 2012; 49: 57-58.

4. Chang SH, Park JH, Kwak JE, Joo M, Kim H, Chi JG, Hong ST, Chai JY. A case of histologically diagnosed tick infestation on the scalp of a Korean child. Korean J Parasitol 2006; 44: 157-161.

5. Kallini JR, Khachemoune A. Ticks and tick bites presenting as "Funny Moles": a review of different presentations and a focus on tick-borne diseases. J Clin Aesthet Dermatol 2017; 10: 46-50.

6. Gökdoğan O, Çakabay T, Baran H, Karabulut B, Tasdemir C, Vatansever Z. Otoacariasis: demographic and clinical outcomes of patients with ticks in the ear canal. Braz J Otorhinolaryngol 2016; 82: 416-421.

7. Barker SC, Walker AR. Ticks of Australia. The species that infest domestic animals and humans. Zootaxa 2014; 3816: 1-144.

8. Shimada Y, Beppu T, Inokuma H, Okuda M, Onishi T. Ixodid tick species recovered from domestic dogs in Japan. Med Vet Entomol 2003; 7: 38-45.

9. Grady CA, McDonald DR, Poppen CF, Pritt BS. Dermacentor tick attached to tympanic membrane. Lancet 2011; 378: 347.

10. Prakairungthong S, Limviriyakul S, Chongvisal S. Temporal bone histopathology case of the month: acute unilateral facial paralysis in child from intra-aural hard tick. Otol Neurotol 2012; 33: 81-82. 